\title{
Comparison of Topical Steroid Drops with Cyclosporine Eye Drops In Epidemic Keratoconjunctivitis
}

\author{
Abdul Rafe ${ }^{1}$, Muhammad Tariq Munawar ${ }^{2}$ \\ ${ }^{1-2}$ Department of Ophthalmology, Combined Military Hospital, Kharian
}

\begin{abstract}
Purpose: To compare the effect of topical steroid with cyclosporine eye drops in recurrence of sub-epithelial corneal infiltrates in epidemic keratoconjunctivitis.

Study Design: Quasi Experimental study.

Place and Duration of Study: CMH Kharian from Jan 2017 to June 2018.

Material and Methods: Eighty eight patients with epidemic keratoconjunctivitis, were divided into two groups. Group A included patients who received topical steroid drops and group B received topical Cyclosporine eye drops. The inclusion criteria comprised of fresh cases of SEls, between the ages of 20 to 50 years, having a vision of $6 / 6$ before the illness. The exclusion criteria included patients suffering from allergic conjunctivitis, ocular surface disease like Sjogren syndrome, corneal ulcer, blepharitis, old corneal opacity, glaucoma and those who had been using steroids in the past e.g. uveitis. The patients were followed up at 2, 4, 8 and 12 weeks. On resolution of sub-epithelial infiltrates (SEls), both regimen were tapered off in next two weeks. The patients were advised to continue monthly follow up for two months to see any recurrence.
\end{abstract}

Results: SEls resolved in both the groups by week 12, however the resolution was slightly quicker in group A. SEls resolved in $84.1 \%$ of cases in group $A$ and in $70.4 \%$ cases in group $B$ at the end of 4 weeks. Recurrence was higher in group A (11.3\%) while it was $4.5 \%$ in group $B$.

Conclusion: Cyclosporine eye drops are a safe and equally effective treatment of epidemic keratoconjunctivitis related SEls, with an added advantage of reduced recurrence rate.

Key Words: Epidemic, Keratoconjunctivitis (EKC), Cornea, Subepithelial Infiltrates, Cyclosporine.

How to Cite this Article: Rafe A, Munawar MT. Comparison of Topical Steroid Drops with Cyclosporine Eye Drops in Epidemic Keratoconjunctivitis. Pak J Ophthalmol. 2020, 36 (4): 445-448.

Doi:

\section{INTRODUCTION}

Epidemic keratoconjunctivitis (EKC) is the most common cause of infectious conjunctivitis worldwide

Correspondence to: Dr. Abdul Rafe

Department of Ophthalmology

Combined Military Hospital, Kharian

Email:mabdulrafe@yahoo.com

Received: 2020

Accepted: 2020 accounting for about $75 \%$ of all cases. ${ }^{1}$ Its morbidity is high in children and immunocompromised patients. ${ }^{2}$ It is caused by adenovirus strains 8,19 and $37 .^{3,4,5,6}$ It has a direct mode of transmission, i.e. by hands, fomites etc. and indirect i.e. by tonometer, eye dropper bottle, slit lamp. ${ }^{3}$ The incubation period is usually 2 to 14 days and the person remains infectious for up to 10 to 14 days after the onset of symptoms. ${ }^{7,8}$ The corneal involvement typically appears after 7 to 10 days of initial presentation in the form of Sub Epithelial Infiltrates (SEIs) in the anterior stroma. ${ }^{9}$ These opacities are pathognomonic of EKC and may persist 
for several weeks to years causing visual deterioration, glare, photophobia and irregular astigmatism. ${ }^{10}$ SEIs represent cellular immune reaction comprising of lymphocytes, macrophages and antigen presenting Langerhans cells. ${ }^{11}$ Although it is a self-limiting disease, but treatment is frequently needed to shorten the duration and relieve the symptoms. ${ }^{12}$ Some Ophthalmologists have used topical steroid eye drops to resolve these lesions with encouraging results, however these drops are associated with increased frequency of recurrence of these lesions. ${ }^{13}$ Cyclosporin eye drops have also shown promising results in resolution of SEIs with an added advantage of reduced chances of recurrence. ${ }^{13}$

This study was done to compare recurrence of SEIs after stopping topical Cyclosporin eye drops versus steroid eye drops.

\section{MATERIAL AND METHODS}

This study was conducted at $\mathrm{CMH}$ Kharian from January 2017 to June 2018, after taking approval from the hospital ethical committee. A total of 88 patients fulfilling the above criteria were included in the study. After taking the informed consent the patients were divided into group A and group Busing nonprobability lottery method. Group A included patients who received topical steroid drops and group $\mathrm{B}$ received topical Cyclosporine A eye drops. The inclusion criteria comprised of fresh cases of SEIs, between the ages of 20 to 50 years, having a vision of $6 / 6$ before the illness. The exclusion criteria was patients suffering from allergic conjunctivitis, ocular surface disease like Sjogren syndrome, corneal ulcer, blepharitis, old corneal opacity, glaucoma and those who had been using steroids in the past. Diagnosis of epidemic keratoconjunctivitis related SEIs was made clinically based on symptoms of glare, photophobia and blurred vision after recent episode of epidemic keratoconjunctivitis. On Slit lamp examination, SEIs were confirmed as greyish white granular sub epithelial lesions with fuzzy borders.

All patients underwent complete ophthalmic examination including visual acuity, tear film assessment, corneal examination and anterior chamber assessment. Intra ocular pressure was measured using air puff tonometer. The corneal sub epithelial opacities were examined under high magnification of slit lamp and counted, and the patients were divided into two groups depending upon the number of SEIs in each cornea, i.e. less than eight SEIs or more than eight SEIs. Patients in group A were started with topical Loteprednol eye drops eight hourly and those of group $\mathrm{B}$ were started with topical Cyclosporine $0.05 \%$ eye drops eight hourly. The patients were followed up at 2 , 4, 8 and 12 weeks. Resolution of SEIs were defined as complete disappearance of these opacities leaving behind clear cornea. On resolution both Cyclosporine and Loteprednol eye drops were tapered off in next two weeks. The patients were advised to continue monthly follow up for next two months, during which they were assessed for any recurrence of corneal SEIs.

Data was recorded in predesigned proforma and SPSS version 21 was used for data analysis. Frequency and percentages were calculated and presented for qualitative data. Student t-test was applied for numerical variables. $\mathrm{P}$ value $\leq 0.05$ was considered as significant.

\section{RESULTS}

A total of 88 patients were included in this study. The demographic details are shown in table 1 which shows the mean age of patients in group A was 30.47 years (20 to 49 years) while it was 29.54 years for group B (20 to 46 years).

Table 1: Demographic characteristics.

\begin{tabular}{lcc}
\hline Age in Years & Group A & Group B \\
\hline $20-30$ & 23 & 25 \\
$31-40$ & 15 & 14 \\
$41-50$ & 6 & 5 \\
Range & 20 to 49 years & 20 to 46 years \\
Mean age & $30.48 \pm 7.53$ & $29.55 \pm 7.17$ \\
No. of Males & 29 & 26 \\
No. of Females & 15 & 18 \\
\hline
\end{tabular}

Table 2: Resolution of Corneal SEIs.

\begin{tabular}{lcc}
\hline Corneal Involvement & Group A (n= 44) & Group B (n= 44) \\
\hline Less than 8 SEIs & $30(68.18 \%)$ & $26(59.09 \%)$ \\
More than 8 SEIs & $14(31.81 \%)$ & $18(40.90 \%)$ \\
Resolution in 2 weeks & $14(31.8 \%)$ & $10(22.7 \%)$ \\
Resolution in 4 weeks & $23(52.2 \%)$ & $21(47.7 \%)$ \\
Resolution in 8 weeks & $4(9.1 \%)$ & $11(25 \%)$ \\
Resolution in 12 weeks & $3(6.8 \%)$ & $2(4.5 \%)$ \\
\hline
\end{tabular}

As is clear from table 2 that SEIs resolved in both the groups by week 12, however the resolution was slightly quicker in group A. In group A $31.8 \%$ of SEIs resolved at the end of second week and another $52.3 \%$ 
by the end of fourth week(total $84.1 \%$ ) whereas $22.7 \%$ resolved at second week and another $47.7 \%$ at the end of fourth week( total $70.4 \%$ ) in group B at the end of same interval.

Table 3: Recurrence of SEIs.

\begin{tabular}{lccc}
\hline & Total Number & Recurrence & P Value \\
\hline Group A & 44 & $5(11.3 \%)$ & \multirow{2}{*}{0.017} \\
Group B & 44 & $2(4.5 \%)$ & \\
\hline
\end{tabular}

\section{DISCUSSION}

Viral Keratoconjunctivitis is a condition that frequently affects the population as an epidemic especially in summers. The human Adenovirus accounts for about $65 \%$ to $90 \%$ of these. ${ }^{14,15,17}$ In addition to its morbidity it also costs in the form of lost productivity and cost of medicines etc. which amounts to 430 million US dollars. ${ }^{16}$ Epidemic Keratoconjunctivitis involves both the conjunctiva and cornea and can cause long lasting morbidity in the form of development of corneal SEIs. Traditionally it had been treated symptomatically with artificial tears, topical antihistamine drops and cold compresses, however the patients demand some treatment to shorten the course of illness and relieve symptoms. In a study conducted by Butt AL, the corneal subepithelial infiltrates lasted for about 45 days causing photophobia, blurring of vision and astigmatism. ${ }^{10}$ It was observed in our study as well where about $70 \%$ to $80 \%$ of SEIs lasted for about 30 days. Some of the ophthalmologists use topical steroids with the aim to shorten the duration and decrease patients discomfort. ${ }^{17}$ These drops make the patients comfortable however they are associated with a higher rate of recurrence of these SEIs and they also increase virus shedding there by prolonging the infectivity periods. ${ }^{18,19}$ Our study also showed that those patients who had used topical steroids showed relatively early resolution as compared to those who used cyclosporine drops, however it was observed that ultimately the corneal lesions resolved in both the groups. There are studies in which there was complete resolution of SEIs in patients who used cyclosporin eye drops. ${ }^{20,21}$ Our study also showed that recurrence of SEIs was lower in patients who used cyclosporine drops being only $4.5 \%$ as compared to $11.7 \%$ in steroid group. Levinger et al in their study, compared the efficacy of cyclosporin eye drops and showed improvement in SEIs in 9 out of 12 patients who had previously used topical steroid drops and were resistant to it. ${ }^{22}$ Similar results were seen in a study by Jeng et al on twelve eyes which have developed SEIs and were responsive to steroids drops but were resistant to tapering. After the initiation of cyclosporin drops, steroids could be successfully tapered without any recurrence. ${ }^{23}$ Romanowisky et al showed in their study that use of topical steroids only improved patients' discomfort, however it did not shorten the course of illness, rather their prolonged use was associated with serious side effects like glaucoma, cataract and corneal thinning. ${ }^{13} \mathrm{We}$ have also found in our study that cyclosporine eye drops are comparable to topical steroid drops in resolving the SEIs but has an added advantage of reduced recurrence rate.

Limitation of our study is the small sample size and single center trial.

\section{CONCLUSION}

Cyclosporine eye drops are a safe and effective treatment of epidemic keratoconjunctivitis related SEIs, with an added advantage of reduced incidence of recurrence.

\section{REFERENCES}

1. Jhanji V, Chan TC, Li EY, Agarwal K, Vajpayee RB. Adenoviral Keratoconjunctivitis. Surv Ophthalmol. 2015; 60 (5): 435-443.

2. Ghebremedhin B. Human Adenovirus: Viral pathogen with increasing importance. Eur $\mathbf{J}$ of Microbial Immuno 1 (BP). 2014; 4: 26-33.

3. Lion T. Adenovirus infection in immunocompetent and immunocompromised patients. Clinical Microbiol Rev. 2014; 27: 441-462.

4. Zhang L, Zhao N, Sha J et al. Virology and epidemiology analysis of global Adeno Virus associated conjunctivitis outbreak 1953-1013. Epidemiol Infect. 2016; 144 (8): 1661-1672.

5. Lee YC, Chan N, Hnng IT et al. Human Adenovirus type 8 epidemic keratoconjunctivitis with large corneal epithelial full layer detachment. An endemic outbreak with uncommon manifestations. Clin. Ophthalmol. 2015; 9: 953-957.

6. Melendez CP, Florentino MM, Martinez IL. Outbreak of epidemic keratoconjunctivitis caused by adenovirus in medical students. Mol Vis 2009; 15: 557562 .

7. Kaufman HE. Adenovirus advances. New diagnostic and therapeutic options. Current Opin in Ophthalmol. 2011; 22: 290-293. 
8. Ford E, Nelson KE, Warren D. Epidemiology of epidemic keratoconjunctivitis. Epidemiol Review. 1987; 9: 244-261.

9. Rajaiya J, Chodosh J. New paradigms in infectious eye disease; adenoviral keratoconjunctivitis. Arch soc Esp Oftalmol 2006; 81: 493-498.

10. Butt Al, Chodosh J. Adenoviral keratoconjunctivitis in a tertiary care eye clinic. Cornea, 2006; 25: 199-202.

11. Kurna SA, Altun A, Oflaz A, Arsan AK. Evaluation of impact of persistent subepithelial corneal infiltrates on the visual performance and corneal optical quality after epidemic keratoconjunctivitis. Acta Ophthalmol. 2015; 93 (4): 377-382.

12. Leanerts L, Naesens L. Antiviral therapy for adenovirus infection. Antiviral Res. 2006; 17: 172-180.

13. Romanowisky EG, Yates KA, Gordon YJ. Short term treatment with a potent topical corticosteroid of an acute ocular adenoviral infection in the New Zealand white rabbit. Cornea; 20: 657-660.

14. Lund E, Stefani FH. Corneal histology after epidemic keratoconjunctivitis. Arch Ophthalmol. 1978; 96: 208588.

15. O' Brien TP, Jeng BH, Mc Donald M, Raizman MB. Acute conjunctivitis truth and misconceptions. Curr Med Res Opin. 2009; 25 (8): 1953-61.

16. Udeh BL, Schneider JE, Obsfeldt RL. Cost effectiveness of a point of care test for adenoviral conjunctivitis. Am J Med Sci. 2008; 336 (3): 254-64.

17. Wilkins MR, Khan S, Bunce C, Khawaja A, Siriwardena D, Larkin DF. A randomized placebocontrolled trial of topical steroids in presumed viral conjunctivitis. Br. J Ophthalmol. 2011; 95: 1299-1303.

18. Clement C, Capriotti JA, Kumar M, et al. Clinical and antiviral efficacy of an ophthalmic formulation of dexamethasone povidone-iodine in a rabbit model of adenoviral keratoconjunctivitis. Inves Sci. 2011; 52 (1): 845-850.

19. Ghanem RC, Vargas JF, Ghanem VC. Tacrolimus for the treatment of subepithelial infiltrates resistant to topical steroids after adenoviral keratoconjunctivitis. Cornea, 2014; 33: 1210-13.

20. Clement C, Coskun E, Jatar MG, Kaydu E, Yayuspayi R, Comez A, et al. Cyclosporin A0.5\% eye drops for the treatment of subepithelial infiltrates after epidemic keratoconjunctivitis. BMC Ophthalmol. 2012; 12: 42.

21. Aydin Kurna S, Altun S, Olfaz A, Karatay Arsan A. Evaluation of the impact of persistent subepithelial corneal infiltration on the visual performance and optical quality after epidemic keratoconjunctivitis. Acta Ophthalmol. 2015; 93: 77-82.

22. Levinger E, Slomovic A, Sansanayndh W, Bahar I, Stomovic AR. Topical treatment with $1 \%$ cyclosporin eye drops for subepithelial infiltrates secondary to adenoviral keratoconjunctivitis. Cornea, 2010; 29: 638640 .

23. Jeng BH, Holsclaw DS. Cyclosporin A $1 \%$ eye drops for the treatment of subepithelial infiltrates after adenoviral keratoconjunctivitis. Cornea, 2011; 30 (9): 958-61.

\section{Author's Designation and Contribution}

Abdul Rafe; Consultant ophthalmologist: Critical appraisal, Drafting and actual write-up of the manuscript.

Muhammad Tariq Munawar; Consultant Ophthalmologist: Concepts, interpretation of data. 